\title{
SOBRE EL TÍTULO DEL LOGISTORICUS GALLUS VEL/AUT FUNDANIUS DE ADMIRANDIS DE MARCO TERENCIO VARRÓN Y LA INSCRIPCIÓN DE THARROS CIL X 7893
}

\author{
Marc Mayer i Olivé \\ Universidad de Barcelona - Institut d'Estudis Catalans \\ mayerolive@yahoo.es
}

\section{RESUMEN}

Se discute en este artículo el título del logistoricus de Varrón que lleva por título Gallus vellaut Fundanius de admirandis y el peso que ha tenido en la biografía del autor la información proporcionada por la inscripción CIL x 7893 y por consiguiente en el título de esta obra, información que se ha tenido en cuenta como un dato cierto por una parte de la tradición filológica a pesar de la cronología mucho más tardía del documento.

PALABRAS CLAVE: Literatura latina, Varrón, epigrafía romana, logistoricus, tradición textual.

ON THE TITLE OF THE LOGISTORICUS GALLUS VEL/AUT FUNDANIUS

DE ADMIRANDIS BY MARCUS TERENTIUS VARRO AND THE INSCRIPTION THARROS CIL X 7893

\section{ABSTRACT}

This paper discusses the title of the Varro's logistoricus entitled Gallus vellaut Fundanius de admirandis and the weight that the information provided by the inscription CIL X 7893 has had in the author's biography and therefore in the title of this work, information that has been taken into account as a fact by part of the philological tradition despite the much later chronology of the document.

KEYwORDS: Roman literature, Varro, Roman epigraphy, logistoricus, textual tradition.

\section{LA CUESTIÓN DEL TÍTULO DEL LOGISTORICUS VARRONIANO}

El título de este logistoricus de Varrón nos es recordado por Nonio Marcelo, con diversas variantes del título que parecería ser en opinión de Lindsay Gallus vel Fundanius de admirandis en cinco ocasiones, que reproducimos en el orden dado por el editor en su índice':

217, 1 (p. 320): Varro Gallo vel Fundanio de miris

205, 33 (p. 303): Varro Gallo aut Fundanio 
71, 21 (p. 99): Varro Gallo vel Fundanio de admirandis rebus

220, 9 (p. 325): Varro Admirandis ${ }^{2}$

218, 16 (p. 322): Varro in Admirandis

Es interesante observar que los manuscritos dan Fundania en el caso de 205, 33 y en el de 71,21 dan para Gallo las variantes callio y gallio. Evidentemente la transmisión de un título de obra con errores de comprensión no tiene nada de sorprendente, pero en este caso nos parece especialmente significativo.

Los gramáticos posteriores que citan esta obra de Varrón lo hacen bajo la forma de un solo nombre Fundanius. Tal es el caso de las dos citas de la Ars grammatica de Flavio Sosipater Carisio: Varro... et in Fundanio, por dos veces con el mismo contenido ${ }^{3}$, además otras cuatro menciones con la indicación in admirandis. Prisciano en sus Institutiones grammaticae libro VII da tan sólo un testimonios: Varro in Fundanio.

Arnobio en su adversus nationes 6, 3 dice: ut tradit in Admirandis Varro ${ }^{6}$. Macrobio por el contrario cita Gallus en sus Saturnalia, 3, 15, 8: M. Varro in libro qui inscribitur Gallus de Admirandis dicit his verbis, con una cita sobre murenas pescadas en Sicilia?.

Servio en su comentario in Georg. III, 113: Varro in libro qui Admirabilium inscribitur y en el comentario de Filargirio al mismo pasaje: Varro in libro qui Mirabilium inscribitur?.

En 1883 L. Havet había notado ya las dificultades de este título en razón de la anteposición de los testimonios de Nonio Marcelo del cognomen al nomen y había propuesto ingeniosamente que debieron existir dos etapas en la composición de este

${ }^{1}$ Es un hecho bien sabido que las referencias a Nonio Marcelo en la edición teubneriana de Lindsay, 1903 están referidas a la edición de Iosephus Mercerus de París 1583 para la primera y 1614 para la segunda; el número del renglón en cambio corresponde a la edición del propio Lindsay. Para facilitar la comprobación hemos hecho seguir a estas siglas un paréntesis con la indicación de las páginas de la edición teubneriana; el fragmento 44 de la edición de Semi, 1965. Para la forma y contenido de los Logistorici, cf. Dahlmann - Heisterhagen, 1957: 5-15 (Heisterhagen) y 15-20 (Dahlmann). Para este logistoricus véase también De Nonno, 2016: 113-138, esp. 130-132 y 138; Piras, 2016: 140-166, esp. 158.

${ }^{2}$ Admirandis es una corrección de Iunius en su edición de Nonio de Amberes 1565 y en sus Animadversiones de Basilea 1556. Los manuscritos dan adnoprandis y noprandis.

${ }^{3}$ Charisius, gramm. I, pp. 61, 8 y 137, 14 Keil. Cf. Keil ,1857.

${ }^{4}$ Charisius, gramm. I, 72, 23; 83, 28; 90, 3 y 131,16 Keil.

${ }^{5}$ Priscianus, gramm. II, p. 331, 19 Keil. Cf. Hertz,1855. Sobre Varrón y Prisciano cf. ahora Rosellini, 2016: 204-221.

${ }^{6}$ Citamos siguiendo la edición de Marchesi, 1934: 310. Sobre Macrobio y Varrón cf. Goldlust, 2016: $167-179$.

${ }^{7}$ Nos servimos de la edición de Marinone, 1967: 430.

${ }^{8}$ Thilo, 1887: 285.

9 Para Filargirio cf. la prudente posición de Hagen, 1867: 703-704; continúa siendo indispensable el trabajo de Funaioli, 1930, en especial el capítulo dedicado a «Le fonti della silloge», pp. 233-270 y pp. 389-390. Véase ahora el sucinto y claro estado de la cuestión en Stok, 2014-2015: 217-240, esp. 217-220. 
título que se reflejaron en su circulación manuscrita: «Varron aurait-il, dans l'une des dernières années de sa vie, substitué le nom de sa femme, ou d'un parent de sa femme, à celui de Cornelius Gallus en disgrâce? $\aleph^{10}$ La hipótesis no deja de ser interesante a la vista de la posibilidad de que pudiera tratarse de Gallus aut Fundania o bien de Gallus aut Fundanius, pero evidentemente resulta muy arriesgada la suposición de que bajo Gallus se ocultara el nombre de Cornelius Gallus el nombre del cual llevaría el logistoricus en su primer momento de circulación antes de un cambio de nombre en función de las circunstancias políticas ${ }^{11}$.

F. Ritschl en 1877 se ocupó de nuevo de la cuestión ${ }^{12}$ y mantiene que a la denominación Gallus del logistoricus debió ser añadido por parte de Varrón el nomen Fundanius por lo común e impreciso en este caso del cognomen y por su voluntad de honrar a su suegro, por que quedaría justificada la secuencia Gallus Fundanius, que recogería así el cognomen de C. Fundanius no conocido por ninguna otra fuente. La partícula disyuntiva en presente en el título conservado por Nonio es atribuida a la negligencia del autor: "perverso loco disiunctiva particula intrusa gemina nomina imperite divulsit» ${ }^{13}$.

Ya en 1910 F. Münzer al redactar el artículo dedicado a C. Fundanius en la $R E$ se hizo eco de lo complicado de esta cuestión onomástica y de la inseguridad de sus elementos ${ }^{14}$.

\section{LA INTERVENCIÓN EN LA CUESTIÓN DEL CONTENIDO DE CIL X 7893}

Un estudio importante de C. Cichorius, fechado en $1922^{15}$, zanjaba la cuestión afirmando que el título del logistoricus varroniano llevaba el nombre del padre de la mujer de su autor, Fundania, C. Fundanius ${ }^{16}$, y que por consiguiente el título debía

${ }^{10}$ Havet, 1883: 176-187 y 193-196, 117.

${ }^{11}$ Zucchelli, 1981: 56-57, ha demostrado con razonamientos cronológicos como esta hipótesis no se puede sostener históricamente.

${ }^{12}$ Ritschl, 1877: 403-418 del vol. III, esp. el capítulo XII: «De M. Terentii Varronis logistoricis libris», concretamente página 406 para el título de la obra donde recoge la totalidad de los testimonios y página 409 con la discusión sobre el título y el nombre del personaje al que se refiere. Además Dahlmann, 1935: cols.1172-1277, esp. cols. 1264-1265. Sobre Varrón en general véase últimamente Leonardis, 2019: 18-24: «Nota biografico-cronologica. "Bimarcus" Terentius Varro: l'uomo dalle molte vite»; anteriormente Marshall, 2016: 180-203, para la cuestión denominada del Bimarcus.

${ }^{13}$ Cf. Ritschl, 1877: 406 del vol. III.

${ }^{14}$ Münzer, 1910: cols. 291-292, esp. col. 292.

${ }^{15}$ Cichorius, 1922: 189-241, el capítulo V dedicado a los «Historische Studien zu Varro» y esp. 205-207.

${ }^{16}$ Sobre el personaje tribuno de la plebe en el 68 a.C. y amigo de Cicerón, cf. MRR II: 138 y 141 nota 8. Además Gruen, 1974: 185, donde indica que en la familia pudo haber senadores desde 


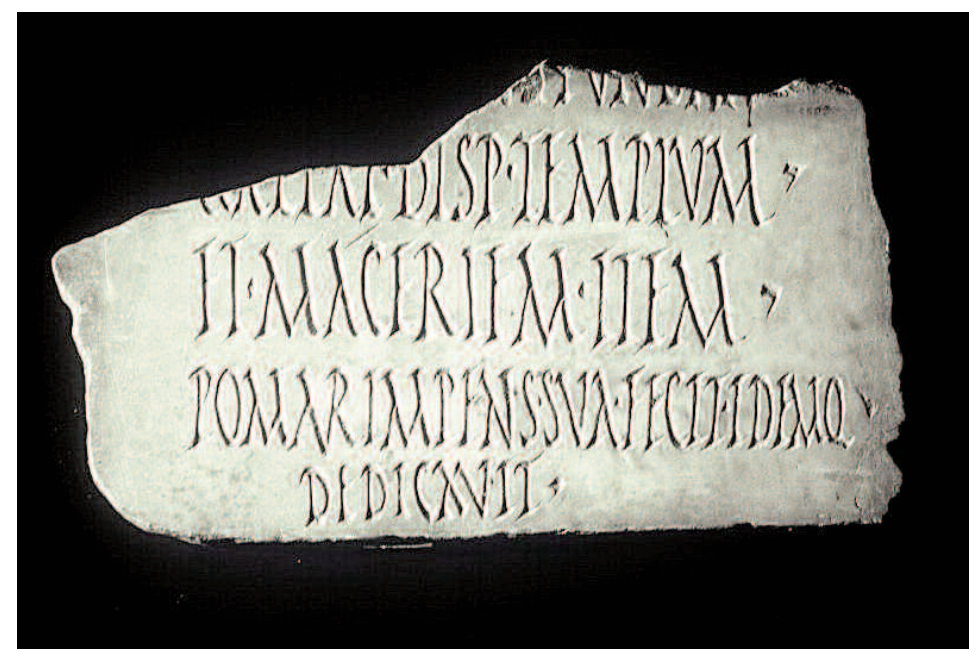

Fig. 1. CIL x 7893, Tharros (fotografia A. Mastino).

de reproducir su nombre completo que por lo tanto sería C. Fundanius Gallus. Se servía para ello de CIL X $7893=I L S 5409^{17}$, donde se menciona el dispensator de una Fundania Galla a la que identifica, a nuestro entender erróneamente, con la mujer de Varrón, argumento que ha sido recogido posteriormente en la literatura científica gracias a la aceptación de dicho razonamiento por parte de J. Heurgon ${ }^{18}$. El texto de la mencionada inscripción reza como sigue (fig. 1):

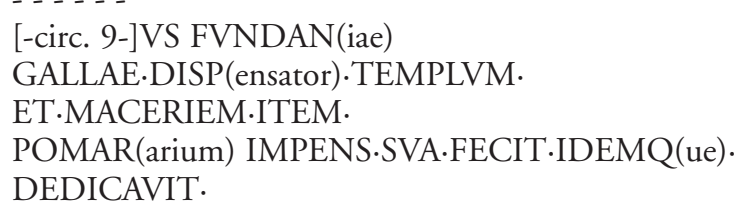

Como podemos ver evidentemente el dispensator depende efectivamente de una Fundania Galla, ha sido también identificada con la esposa de Varrón en

la época silana o incluso mucho antes; en página 516, lo incluye en la lista de tribunos de la plebe de familia senatorial; cf. la página 527, para la defensa de C. Fundanius por parte de Cicerón antes del 64, Comm. petit. 19, quizás se trató de un caso de repetundae. Quint. inst. 1, 4, 14, se refiere al Pro Fundanio de Cicerón: nam contra Graeci adspirare fsolent, ut pro Fundanio Cicero testem, qui prima eius litteram dicere non possit, inridet, citamos siguiendo la edición de la Collection des Universités de France de Cousin, 1975: 82.

${ }^{17}$ Además Corda, 2014: 90, núm. 373; Porrà, 2002: 949, núm. 756. EDCS-22500068, que la data erróneamente entre el 27 y el 14 a.C.

${ }^{18}$ En su edición del De re rustica en la Collection des Universités de France, Heurgon, 1978: 92. 
la bibliografía específica dedicada a Sardinia ${ }^{19}$, no haremos un comentario epigráfico detenido, pero en este punto conviene hacer algunas precisiones desde este punto de vista. En primer lugar, hemos de señalar que el VS inicial no es seguro, pero sí altamente probable, y que la letra, como ya hemos indicado, es una capital que no dudaríamos en clasificar como derivada de la libraria del siglo II d.C. La A del renglón 3 lleva un apex. En cuanto al formulario nos hallamos ante la secuencia templum, maceries $\mathrm{y}$ pomarium, que resulta inédita en esta forma trimembre, aunque en cambio la secuencia templum y maceries está bien documentada. La combinación de pomarium con maceries o maceria también es conocida en el caso de recintos sepulcrales. Resulta especialmente notable el ejemplo para este tipo de sepulturas de la tumba descrita en el llamado testamentum Lingonis, CIL XIII 5708 = ILS 8379, de Langres, Andematum, donde se mencionan saepta et pomaria. La dedicación de una sepultura sirviéndose de las formas fecit y dedicavit es frecuente ${ }^{20}$. Evidentemente no parece tratarse del recinto funerario de Fundania Galla, sino de alguna persona o grupo de personas con lazos de relación con el dispensator. La figura de la mujer de Varrón pesa en todas las identificaciones. Es especialmente notable el trabajo de R. Zucca, que supone que la mujer de Varrón, Fundania Galla, en el período augusteo a través de su dispensator habría hecho construir un templum et maceriem item pomar(ium), aunque advierte prudentemente que la atribución no es suya y que sigue una tradición ya establecida ${ }^{21}$.

De forma más general podemos concluir que la paleografía de la inscripción parece, como puede comprobarse, desmentir esta atribución e identificación directa tal como insistiremos más adelante.

Más recientemente B. Zucchelli ${ }^{22}$, 1981, usa de nuevo el epígrafe al que nos hemos referido como evidencia de un documento independiente del texto del propio Varrón que supondría la existencia de un Fundanius Gallus, que identifica con el Fundanius del De re rustica, que resultaría así ser el padre de Fundania, mujer de Varrón. La variedad de formas de citar el logistoricus y consecuentemente el personaje que hallamos en Nonio serían producto de la imprecisión del lexicógrafo y precisa: "col fatto che dei due nomi soltanto il primo, e precisamente il cognomen, come negli altri logistorici, sarà apparso nel titulus esterno, mentre l'altro sarà stato scritto all'interno del rotolo con il sottotitolo", continúa Zucchelli indicando que un tema semejante

${ }^{19}$ Sigue esta hipótesis la historiografía sarda posterior: Zucca, 1994: 857-935, esp. 892 y 894, núm. 85 y lám. XII, 85; de nuevo Zucca, 2005: 263; también Mastino, 2005: 182, donde supone que se trata de la mujer de Varrón, siguiendo la communis opinio, que también había hecho suya P. Meloni en 1975, cf. Meloni, 2012: 185-186.

${ }^{20}$ Sirvan de ejemplo entre centenares de otros: CIL VI 12562 y CIL VII 9077 y 9142.

${ }^{21}$ Zucca, 1994: 892 y nota 196: «L'dentificazione tra Fundan(ia) Galla dell'iscrizione tharrense e l'omonima moglie di M. Terenzio Varrone è stata proposta da C. Cichorius, Historische Studien zu Varro, "Römische Studien" 1961, pp. 103-104; cfr. Meloni, Sardegna, p. 291; J. Heurgon, Varron (Les Belles Lettres) p. 92».

${ }^{22}$ Zucchelli, 1981: 56-57. 
a los $\theta \alpha v \mu \alpha ́ \sigma 1 \alpha$ peripatéticos convendría a un propietario agrícola importante, pero no de alta cultura, como sería C. Fundanius, razón por la cual le habría dedicado esta obra su yerno Varrón.

La edición de las Saturae Menippeae de W. A. Krenkel, 2002, da también como hecho seguro a partir de la inscripción citada que el nombre de la mujer de Varrón sea Fundania Galla y que su padre llevara el nombre de Fundanius Gallus y que el logistoricus se titulara Gallus Fundanius de admirandis al comentar la sátira De officio mariti ${ }^{23}$.

Ha vuelto sobre el tema aún más recientemente Y. Lehmann ${ }^{24}$, que cree que el título del logistoricus sería efectivamente Gallus Fundanius de admirandis y también que sin duda estaría dedicado a C. Fundanius Gallus, padre de la mujer de Varrón y personaje principal de la obra, al tiempo que intenta precisar el contenido de los admiranda o miracula que recogía Varrón en boca de Fundanius que parece depender a su vez de los Sasernae $e^{25}$, tomando como referencia los pasajes del De re rustica en que está mencionado ${ }^{26}$ para destacar sus intereses que encuentra a continuación reflejados en los comentaristas tardíos de Virgilio que citan un liber Admirabilium de Varrón (Servio ad Georg. III, 113) y un liber Mirabilium (Filargirio, comentario al mismo verso, ad Georg. III, 113) ${ }^{27}$.

\section{UNA NUEVA CONSIDERACIÓN DEL PROBLEMA}

La posibilidad que proponemos considerar en estas breves páginas es considerar el título del logistoricus por sí mismo y en el marco de la obra varroniana, sin introducir el documento epigráfico sardo al que nos hemos referido ${ }^{28}$. No cabe duda de que el hacerlo de esta manera no entorpece la interpretación del título como referido al padre de Fundania, esposa de Varrón, sin necesidad de considerar para ella un cognomen, probable pero no probado Galla, teniendo en cuenta además que el conocimiento que tenemos de ella depende fundamentalmente del De re rustica de Varrón que la cita en tres ocasiones ${ }^{29}$ y no da en ningún caso en dicha obra este

${ }^{23}$ Krenkel, 2002:141. Mucho más prudente se mostró Della Corte, 1954: 230, donde se refiere a Fundanio como suegro de Varrón sin más precisiones. No entra en la cuestión el comentario de Cèbe, 1975: 354-364, que sólo se refiere a las ideas de Varrón sobre el matrimonio sin introducir circunstancias personales del autor, cf. esp. Cèbe, 1972: 41-44, para las ideas de Varrón sobre esta cuestión y el amor.

${ }^{24}$ Lehmann, 2006 : 553-557.

${ }^{25}$ Lehmann, 2006 : 554-555.

${ }^{26}$ Res rustica, 1, 2, 5; 1, 2, 15; 1,2, 18-19

${ }^{27}$ Lehmann 2006: 556-557. Cf. nuestras notas 8 y 9.

${ }^{28}$ Dahlmann, 1935: cols. 1173-1181 para la biografía y col. 1185 para la dedicatoria de su obra sobre la agricultura a su esposa Fundania, que poseía un fundus in Sabinis.

${ }^{29}$ Res rustica 1, 1, 1 en el encabezamiento de la obra que le dedica; en el párrafo 6 del prefacio del libro II en que recuerda al dedicatario, Turranius Niger, que el libro anterior había sido dedicado a su esposa Fundania, en razón del fundus de ésta, y por último en 3, 1, 9, en que vuelve a recordarla 
cognomen ni a ella ni a su padre. El nombre de C. Fundanius aparece con mayor frecuencia en el De re rustica, pero se limita únicamente al libro primero dedicado a la agricultura ${ }^{30}$.

Nuestra propuesta por otra parte nos permite continuar pensando en que el título del logistoricus y la conjunción disyuntiva vel, que parece a todas luces contener, pueden reflejar un supernomen o signum ${ }^{31}$ de Fundanius más que un cognomen, el cual podría estar vinculado a una origo o bien incluso a una experiencia personal como sucede en ocasiones con los agnomina honoris que no parece que tengan que ser considerados como una posibilidad en este caso. El carácter elogioso de las menciones de Varrón a Fundanius queda fuera de toda duda, el mismo logistoricus tendría también un carácter de laudatio ${ }^{32}$.

Podrá decirse que la inscripción de Cerdeña puede estar relacionada con esta familia y que los Fundanii no son muchos. Nada tendríamos que objetar a esta probabilidad, si se tiene en cuenta la distancia cronológica entre la obra varroniana y el período imperial posiblemente antonino que parece reflejar la facies de dicha inscripción. El nombre Fundania Galla sería en este período más fácilmente relacionable con los Fundanii que van a convergir en la familia de Antonino Pío ${ }^{33}$, que con la pervivencia de una familia republicana de la que pudieron muy bien descender.

Sabemos que M. Annius Verus, cónsul por tercera vez en el año 126 d.C. era el proavus paternus de Marco Aurelio, y padre de M. Annius Verus, hermano de Annia Galeria Faustina, y padre a su vez de Marco Aurelio y de Annia Cornificia Faustina,

junto con Turranius como destinatarios de la dedicatoria de los libros anteriores. Hay que señalar de todas maneras en 1, 1, 4 dice a su esposa: Quocirca scribam tibi tres libros indices ad quos revertare, si qua in re quaeres quem ad modum quidque te in colendo oporteat facere, citamos siguiendo la edición en la Collection des Universités de France de Heurgon, 1978: 8. El hecho de que cambien de destinatario los dos libros siguientes hizo pensar a Francken1900, 281-297 y 422-435, esp. 294, que Fundania habría muerto o habría mediado un divorcio antes de la publicación de los siguientes volúmenes: «Nisi Varronem inhumanitatis et feritatis incusare velimus statuendum erit Fundaniam post primum librum scriptum et traditum decessisse aut certe more illa aetate Romanis recepto in alius mariti penates migrasse», evidentemente se trata de un elemento probatorio muy débil, aunque posible.

${ }^{30}$ Res rustica 1, 2, 1 donde se refiere al mismo como socer, 1, 2, 5, donde reproduce una intervención de Fundanius que se extiende hasta 1, 2, 10;1, 2, 15, una nueva intervención de Fundanius; $1,2,18$ otra que llega a 1,$20 ; 1,2,26$, de nuevo una intervención breve como también se da en 1,2 , $27 ; 1,12,2$, donde se pone una pregunta; $1,13,6$ da una opinión moralizante sobre las villae rusticae opuestas a la de recreo que se alarga hasta 1,13, 7 y finalmente 1, 26, donde otro personaje Agrius se dirige en su diálogo a Fundanius.

${ }^{31}$ De forma general Kajanto, 1966.

${ }^{32}$ Cf. para la relación entre logistorici y laudationes, Gwyn Morgan, 1974: 117-128, esp. 117-122.

${ }^{33}$ Cf. FOS: 340-341, núms. 393-394, hay que tener en cuenta sin embargo que la Fundania propuesta en núm. 393 es fruto de una deducción prosopográfica, que la supone hija de L. Fundanius Lamia Aelianus, PIR A 204, y casada con M. Annius Libo, PIR² A 667, hermano de Faustina maior y padre de Annia Fundania Faustina, cf. FOS: 76-77, núm. 60, prima de Marco Aurelio, que sería a su vez madre de T. Fundanius Vitrasius Pollio, PIR ${ }^{2}$ F 395. 
que se casó con un Ummidius Quadratus ${ }^{34}$. Debemos aquí hacer hincapié en que Annia Galeria Faustina es Faustina ${ }^{35}$ y en el hecho de que los Annios Veros están emparentados con Galeria Fundana, esposa de Vitelio ${ }^{36}$, como se puede deducir a partir del nombre de Annia Galeria Faustina, la mujer de Antonino Pío, y vinculada a los Fundanii del siglo II como propusó convincentemente sir Ronald Syme ${ }^{37}$. Hay que insistir además en el hecho de que Annia Fundania Faustina es consobrina de Marco Aurelio y de Faustina II ${ }^{38}$. No queremos suponer con ello que la Fundania Galla frente a la cual nos encontramos pueda entroncarse directamente con la casa imperial antonina, pero debemos convenir en que si quisiéramos dar crédito a esta posibilidad no nos faltarían argumentos, aunque evidentemente ninguno decisivo.

Volviendo de nuevo a la cuestión del título del logistoricus que nos ocupa, veremos que el praenomen de C. Fundanius, padre de Fundania, mujer de Varrón, parece conducir al personaje a un parentesco con los otros C. Fundanii conocidos $^{39}$, para los que no tenemos en ningún caso una presencia de un cognomen Gallus.

Como corolario para las dos cuestiones tratadas podemos decir que aún en el caso de que se quiera prescindir del velo del aut en el título del logistoricus varroniano y se quiera ver una mención de la estructura onomástica de un C. Fundanius Gallus, nada hace suponer que la mujer de Varrón llevara por nombre además del de Fundania el cognomen Galla y que además estuviera documentada en Sardinia como propietaria rural. Una supuesta coincidencia onomástica, combinada con la ausencia de un examen directo de la realidad epigráfica considerada, ha llevado a encabalgar conjeturas, que han conducido a extraer unas conclusiones que a partir del examen objetivo de los documentos parecen no ser posibles, aunque hayan sido generalmente aceptadas.

Hemos presentado en suma unas pequeñas notas entre filología y epigrafía, textos literarios e inscripciones, que esperamos que serán gratos a nuestro homenajeado, cuya obra muestra su competencia en ambos campos.

\section{REFERENCIAS BIBLIOGRÁFICAS}

CÈBE, J.-P. (1972: Varron, Satires Ménippées. Édition, traduction et commentaire, 1 (CEFR, 9), Roma. CÈBE, J.-P. (1975): Varron, Satires Ménippées. Édition, traduction et commentaire, 3 (Caprinum proelium - Endymiones) (CEFR, 9), Roma.

${ }^{34}$ Cf. FOS:73-74, núm. 57.

${ }^{35}$ FOS :78-79, núm. 62.

${ }^{36}$ Cf. FOS: 344-346, núm. 399.

${ }^{37}$ Syme, 1982: 460-473, esp. 475, nota 8.

${ }^{38}$ FOS: 80-81, núm. 63.

${ }^{39}$ MRR II: 568, el único cognomen documentado es Fundulus. 
CICHORIUS, C. (1922): Römische Studien. Historisches epigraphisches literargeschichtliches aus vier Jahrhunderten Roms, Leipzig, Berlin [reimpr. Darmstadt 1961].

Corda, A. M. (2014): Concordanze delle iscrizioni latine della Sardegna. Edizione dei testi e indice dei vocaboli, Ortocesus.

CousIn, J. (ed.) (1975): Quintilien, Institution oratoire, tome I, livre I, Paris.

Dahlmann, H. (1935): «84) M. Terentius Varro», RE Supp. VI: cols. 1172-1277, Stuttgart.

Dahlmann, H. - Heisterhagen, R. (1957): Varronische Studien I. Zu den Logistorici, Wiesbaden (= Akad. d. Wiss. und d. Lit. Abh. d. geistes- und sozialwissenschaftlichen Klasse, 1957, nr. 4, pp. 127-174).

Della CoRTE, F. (1954): Varrone il terzo gran lume romano, Genova.

De Nonno, M. (2016): «Forme e modi della presenza di Varrone nei grammatici latini, tracce di dottrina e documenti linguistici», Res publica litterarum 39 (n.s. 19): 113-138.

$F O S=$ RAEPSAET-CHARLIER, M.-Th. (1987): Prosopographie des femmes de l'ordre sénatorial (Ir-II siècles) (Académie Royale de Belgique. Classe des Lettres. Fonds René Draguet, t. Iv), Louvain.

FranCKen, C. M. (1900): «Varroniana», Mnemosyne 28: 281-297 y 422-435.

Funaioli, G. (1930): Esegesi virgiliana antica. Prolegomeni alla edizione del commento di Giunio Filargirio e di Tito Gallo (Pubblicazioni della Università Cattolica del Sacro Cuore, ser. IV, IX), Milano.

GoldLusT, B. (2016): «Reconstructing the Republic: imperial citing practices of Varro and the case of Macrobius' Saturnalia», Res publica litterarum 39 (n.s. 19): 167-179.

Gruen, E.S. (1974): The Last Generation of the Roman Republic, Berkeley, Los Angeles.

GwYn Morgan, M. (1974): «Three Notes in Varro's Logistorici», Museum Helveticum 31: 117-128.

Hagen, H (1867): Scholia Bernensia ad Vergili Bucolica atque Georgica, Leipzig.

HAVET, L. (1883): «Varroniana», RPh VII: 176-187 y 193-196.

HerTZ, M. (1855): Prisciani grammatici Caesariensis institutionum grammaticarum libri XVIII, vol. I, Leipzig [corresponde a H. KeIL, Grammatici Latini, vol. II, Prisciani institutionum grammaticarum libri I-XII].

Heurgon, J. (1978): Varron, Économie rurale, livre premier, Paris.

Kajanto, I. (1966): Supernomina. A Study in Latin Epigraphy (Commentationes humanarum litterarum. Societas scientiarum Fennica, 40/1), Helsinki-Helsingfors.

KeIL, H. (1857): Grammatici Latini, vol. I, Flavii Sosipatri Charisii artis grammaticae libri V, Diomedis artis grammaticae libri III ex Charisii arte grammatica excerpta, Leipzig.

Krenkel, W. A. (ed.) (2002): Marcus Terentius Varro, Saturae Menippeae, vol. I (Subsidia Classica, 6), St. Katharinen.

LeHmanN, Y. (2006): «Le merveilleux scientifique dans le Logistoricus Gallus Fundanius de Admirandis de Varron», en J. ChampeauX - M. CHASSIGNET (eds.), Aere perennius en hommage à Hubert Zehnacker, Paris (Roma antiqua), pp. 553-557.

LEONARDIS, I. (2019): Varrone, unus scilicet antiquorum hominum senso del passato e prattica anticuaria (Biblioteca Athenaeum, 62), Bari.

LINDSAY, W. M. (ed.) (1903): Nonii Marcelli de compendiosa doctrina libros XX, Leipzig.

MARCHESI, C. (ed.) (1934): Arnobii adversus nationes (Corpus scriptorum Latinorum Paravianum, 62), Torino. 
Marinone, N. (ed.) (1967): I Saturnali di Macrobio Teodosio (Classici Latini), Torino.

Mastino, A. (2005): Storia della Sardegna antica (La Sardegna e la sua storia, II), Nuoro.

Meloni, P. (2012): La Sardegna romana, Nuoro [reimpr. de la 2a edición de Sassari 1990, la primera edición es de 1975].

MRR II = BROUGHTON, T. R. S. (1968): The Magistrates of The Roman Republic, vol. II (Philological Monographs published by the American Philological Association, XV, II), Cleveland, Ohio 1968 [reimpr. de la primera ed. 1952].

MÜNZER, F. (1910): «1) C. Fundanius», RE VII.1: cols. 291-292, Stuttgart.

PIRAS, G. (2016): «Sulle citazioni di Varrone in Nonio. Alcune osservazioni», Res publica litterarum 39 (n.s. 19): 140-166.

PORRÀ, F. (2002): Catalogo P.E.T.R.A.E. delle iscrizioni latine della Sardegna: versione preliminare, Cagliari.

Ritschl, F. (1877): Opuscula Philologica. Volumen III: ad litteras Latinas spectantia, Leipzig.

Rosellini, M. (2016): «Varrone in Prisciano. Un interlocutore mancato», Res publica litterarum 39 (n.s. 19): 204-221.

SEMI, F. (ed.) (1965): M. Terentius Varro, Fragmenta operum de grammatica litteris philosophia scientiis; Logistoricon libri; Antiquitates, vol. 2 (Scriptorum Romanorum quae extant omnia, 42), Venezia.

STOK, F. (2014-2015): «"Philargyrius” biografo di Virgilio», RET 4: 217-240.

SYME, R. (1982): «Partisans of Galba», Historia 31: 460-473.

THILO, G. (ed.) (1887): Servii grammatici qui feruntur in Vergilii Bucolica et Georgica commentarii, Leipzig.

ZuCCA, R. (1994): «Il decoro urbano delle civitates Sardiniae et Corsicae: il contributo delle fonti letterarie ed epigrafiche», en A. Mastino, P. RUGGeri (eds.), L'Africa Romana. Atti del X convegno di studio, Oristano, 11-13 dicembre 1992 (Collana del Dipartimento di Storia dell'Università degli Studi di Sassari, 25), Sassari, pp. 857-935.

Zucca, R. (2005): «Gli oppida e i populi della Sardinia», en A. Mastino, Storia della Sardegna antica (La Sardegna e la sua storia, II), Nuoro, pp. 205-332.

Zucchelli, B. (1981): Varro logistoricus. Studio letterario e prosopografico (Università di Parma. Istituto di lingua e letteratura latina, 5), Parma. 